6. Tian W, Yakirevich E, Matoso A, et al. IgG4+ plasma cells in sclerosing variant of mucoepidermoid carcinoma. Am J Surg Pathol 2012; 36: $973-9$.

7. Ota M, Katsuyama Y, Hamano H, et al. Two critical genes (HLA-DRB1 and $\mathrm{ABCF} 1$ ) in the HLA region are associated with the susceptibility to autoimmune pancreatitis. Immunogenetics 2007; 59: 45-52.

8. Ohta N, Makihara S, Okano M, et al. Roles of IL-17, Th1, and Tc1 cells in patients with IgG4-related sclerosing sialadenitis. Laryngoscope 2012; 122: $2169-74$.

9. Zen Y, Fujii T, Harada K, et al. Th2 and regulatory immune reactions are increased in immunoglobin G4-related sclerosing pancreatitis and chol angitis. Hepatology 2007; 45: 1538-46.

DOI: http://dx.doi.org/10.1016/j.pathol.2017.07.009

\section{Multi-organ vaso-occlusive disease: Buerger's or Kohlmeier-Degos disease?}

Sir,

A multi-organ vaso-occlusive disorder can be due to vasculitis, atheroembolic diseases, or thrombotic conditions such as antiphospholipid syndrome. Multisystem Buerger's disease and Kohlmeier-Degos disease are two rarer conditions that should be considered after exclusion of more common disorders.

A 33-year-old man presented in November 2013 with headache, nausea and vomiting. His medical history included hypertension, left second toe amputation for ischaemia 4 months prior and smoking (10-15 cigarettes a day). His physical examination was unremarkable apart from hypertension at $170 / 100 \mathrm{mmHg}$. He had developed acute kidney injury with a serum creatinine of $731 \mu \mathrm{mol} / \mathrm{L}$ (eGFR $8 \mathrm{~mL} /$ min) from a baseline of $85 \mu \mathrm{mol} / \mathrm{L} 5$ months prior; urea was $38.8 \mathrm{mmol} / \mathrm{L}$. His haemoglobin and platelet count were within the normal range, and he did not have schistocytes on his blood film. His autoantibody panel was negative. Examination of urine revealed $55 \times 10^{6} / \mathrm{L}$ glomerular red cells, and $1.2 \mathrm{~g}$ per day of proteinuria. A renal ultrasound was unremarkable and renal artery waveforms were normal.

A renal biopsy (Fig. 1) revealed one glomerulus with segmental intraluminal thrombi, and a large artery with intraluminal thrombus and florid re-organisation of the vessel wall. Although the associated internal elastic lamina was disrupted, it was not extensively fragmented or duplicated, as seen in vasculitis. Arterioles and small arteries were normal. He was given a provisional diagnosis of accelerated hypertensive nephropathy despite not having the characteristic features of malignant hypertension on renal biopsy. His renal function did not recover and peritoneal dialysis was commenced.

In October 2015 he suffered critical ischaemia of the left foot despite normal distal pulses and Doppler ultrasonography demonstrating good three vessel run-off without stenosis. He underwent a below-knee amputation. Histology demonstrated segmental occlusive thrombotic changes in the arteries in keeping with Buerger's disease (Fig. 2). Of note, he continued to smoke cigarettes, a well-known risk factor for Buerger's disease. However, he did not have features of thrombophlebitis migrans, which could be associated with Buerger's disease. He developed urinary retention during this admission and a Candida albicans urinary tract infection
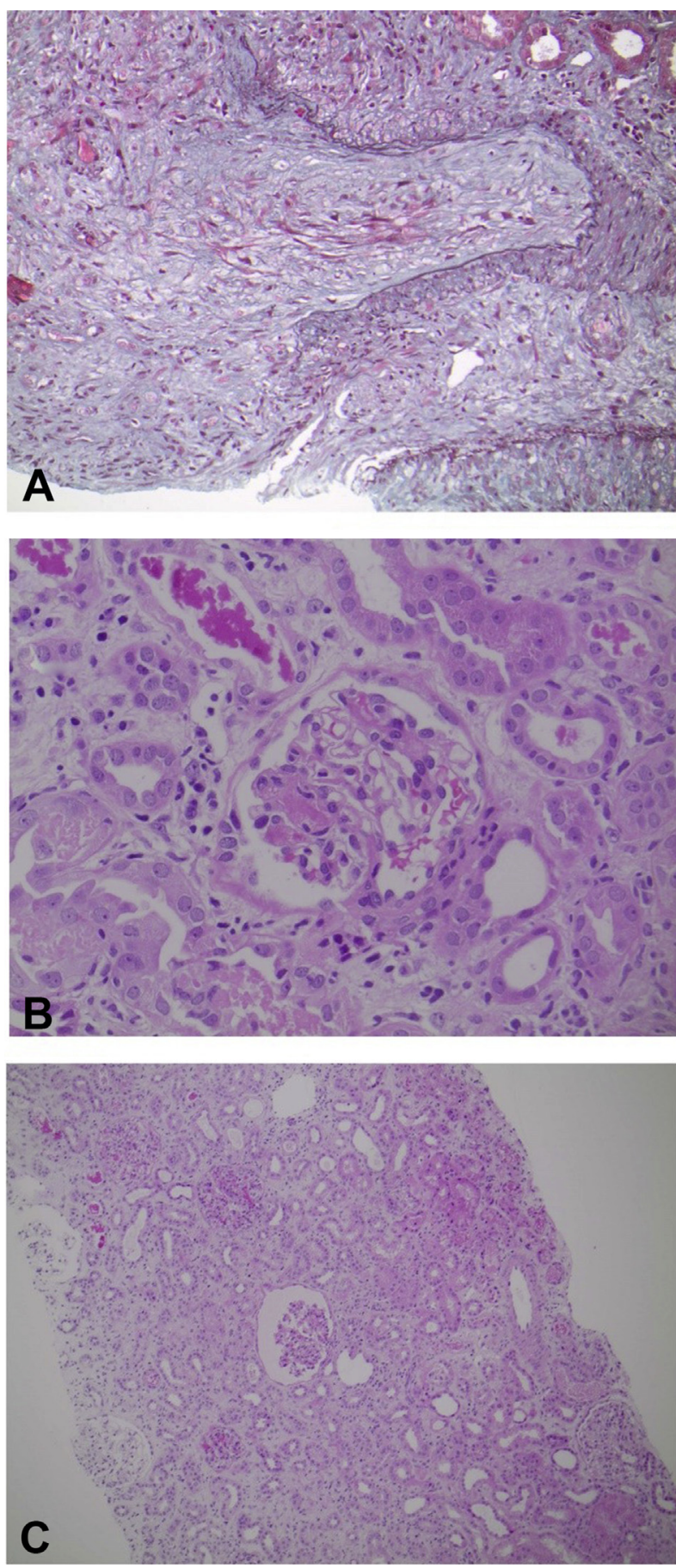

Fig. 1 Kidney biopsy demonstrating (A) an arcuate artery with organising thrombus and disrupted elastic lamina, (B) a glomerulus with segmental intraluminal thrombi, and (C) an ischaemic glomerulus and interstitial oedema with patchy infiltrate of lymphocytes, histiocytes and a few eosinophils.

managed with fluconazole. In the ensuing 8 months he had recurrent episodes of Candida urinary tract infections, treated with oral fluconazole and amphotericin bladder washouts.

In August 2016, he developed Candida peritonitis necessitating removal of the Tenckhoff catheter. At surgery he was noted to have a small bowel perforation and a small bowel resection was performed. Histopathology demonstrated mucosal ulcers and abnormalities in the arteries of the bowel wall and surrounding fat. Arterial changes included recent thrombosis in addition to chronic arterial lesions with 

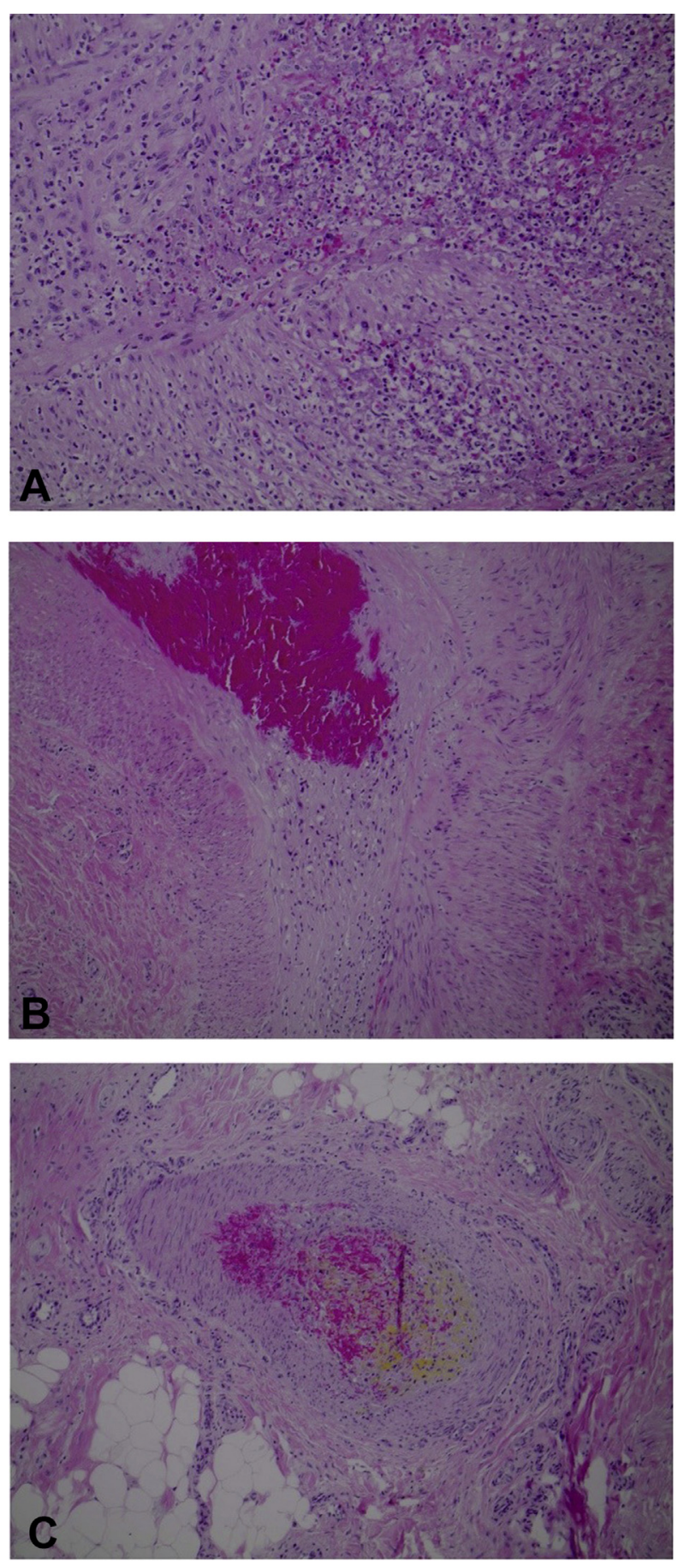

Fig. 2 Left leg amputation histology showing (A) an acutely inflamed artery with transmural neutrophils and lymphocytes, (B) an artery showing both acute thrombosis and a chronic organised thrombus with mononuclear cells, and $(\mathrm{C})$ an artery showing luminal organising thrombus with adjacent haemosiderin pigment and transmural inflammation.

organisation and recanalising thrombi with collections of haemosiderin laden macrophages and intimal and medial fibrosis. Some arteries showed marked luminal narrowing or occlusion with an infiltrate of mixed inflammatory cells (Fig. 3). In retrospect, he most likely first developed ischaemic colitis, which was complicated by bowel perforation and candida peritonitis. The admission was complicated by gastrointestinal haemorrhage due to gastritis and duodenitis, and a myocardial infarct. Of note, coronary angiography revealed smooth coronary arteries.

He has since recovered from his multiple complications and currently receives outpatient haemodialysis. He has stopped smoking and hopes to recover sufficiently to undertake live donor renal transplantation from his father.

Buerger's disease, also known as thromboangiitis obliterans, is an episodic, segmental, inflammatory vasoocclusive disease, most commonly affecting the young male Asian population who do not have other atherosclerotic risk factors apart from smoking. ${ }^{1}$ It is characterised by thrombotic occlusions in the small to medium sized arteries and veins in the extremities. Histologically, the acute occlusions are accompanied with neutrophilic infiltration and occasional microabscesses. Chronic lesions contain organising or organised thrombi with predominantly mononuclear infiltrates and multinucleated giant cells. Even though the normal structure of the vessel typically remains unaffected in Buerger's disease, unlike in vasculitis, ${ }^{3}$ some cases are associated with disruption of the internal elastic lamina. ${ }^{2}$ Visceral vessel involvement is rare, but there are reports of mesenteric, coronary and cerebral vessels being affected. ${ }^{4}$

The aetiology of Buerger's disease remains uncertain. It is unclear whether the initial insult is thrombosis or inflammation, and the pathophysiology of this condition likely involves an interplay between host susceptibility and a trigger such as cigarette smoking or infectious agents, exacerbated by psychological stresses. ${ }^{3}$ There is also an association with low socioeconomic status.

A second pathologist reviewed all the histological specimens from this patient and proposed Kohlmeier-Degos disease as an alternative diagnosis. Also known as malignant atrophic papulosis, this condition is rare with only about 300 reported cases. ${ }^{5}$ There is a benign form of the condition, with only cutaneous involvement, which manifests as large papules with an erythematous rim and an atrophic porcelainwhite centre. ${ }^{6}$ However, the systemic variant has a poorer prognosis. It is characterised by thrombotic occlusion of the small arteries and arterioles, and occasionally veins, accompanied by endothelial swelling and intimal proliferation and mononuclear cell infiltration, leading to tissue necrosis and infarction. ${ }^{5}$ The most commonly affected organs are the gastrointestinal tract $(50 \%)$ and the central nervous system $(20 \%){ }^{6}$ The aetiology for Kohlmeier-Degos disease is also unknown.

Histopathological changes from both Buerger's disease and Kohlmeier-Degos disease can be indistinguishable, with vascular thrombosis of varying chronicity associated with an inflammatory infiltrate and intimal hyperplasia. In our case, the lack of typical cutaneous lesions might argue against Kohlmeier-Degos disease. However, Buerger's disease is less frequently associated with gastrointestinal tract or other visceral involvement.

Renal involvement in both conditions has rarely been reported in the literature. Barcelli et al. in 1981 described a similar case of a 43-year-old man who developed renal failure and an ischaemic limb and ultimately died from mesenteric ischaemia. ${ }^{8}$ His initial renal biopsy showed arteriolar lesions with endothelial swelling, intimal proliferation and medial hypertrophy. Most of his glomeruli displayed ischaemic changes. It was unclear if there were thrombi present. However, his post-mortem demonstrated occlusion of mesenteric 

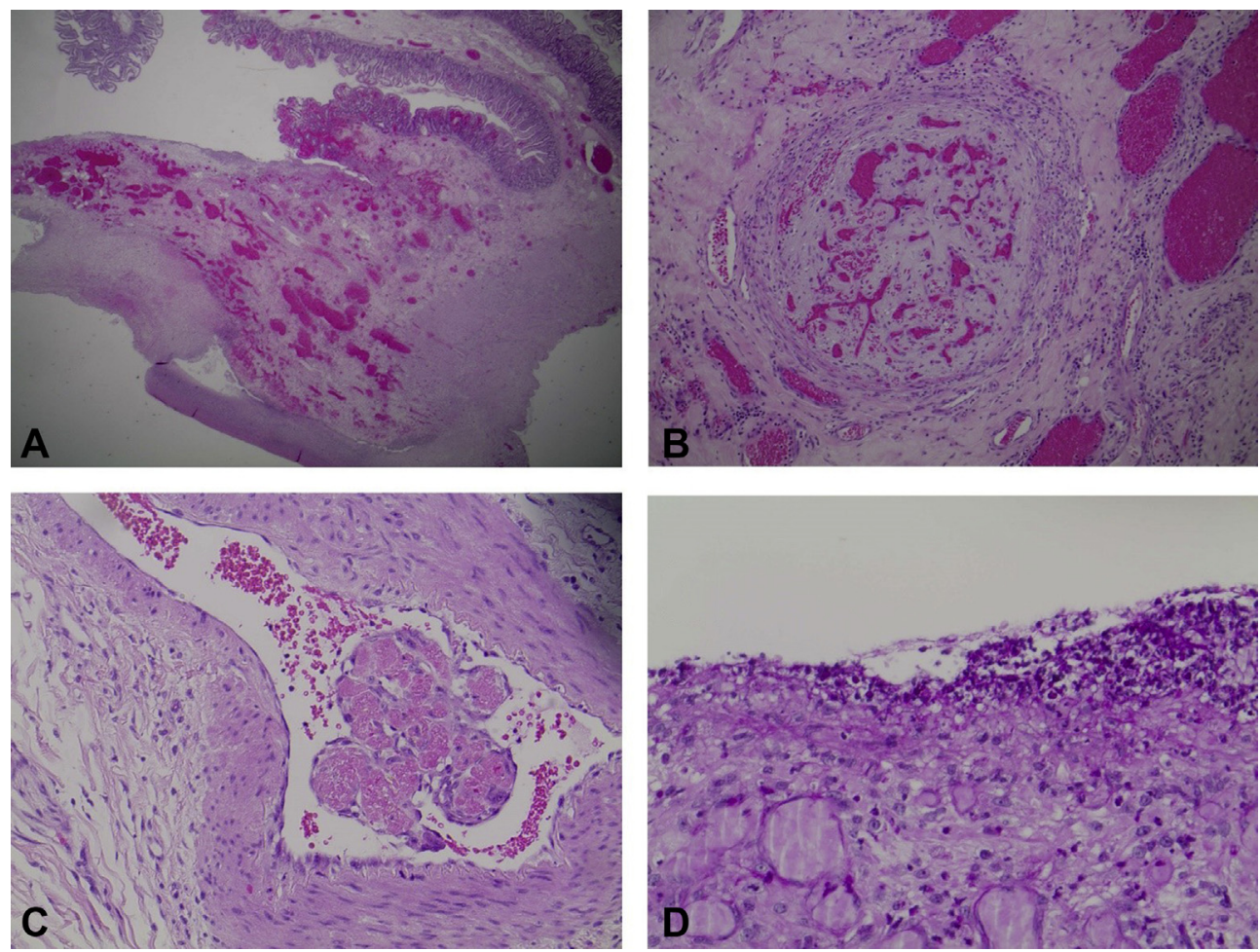

Fig. 3 Bowel resection histology showing (A) ulceration and necrosis of the bowel wall with associated perforation, acute inflammation and granulation tissue, (B) recanalising thrombus in an artery, (C) an artery with organising luminal fibrin thrombus and endothelial proliferation but no mural inflammation, and (D) luminal Candida on PAS stain.

and renal arteries by thrombus and intimal proliferation. The author concluded that the clinical picture was most in keeping with Kohlmeier-Degos disease. ${ }^{8}$ Another similar case was reported by Björck et al. in 1984, of a 45-year-old man with renal failure due to arterial occlusion from concentric intimal proliferation, again consistent with Kohlmeier-Degos disease. He died 2 months later from gastrointestinal bleeding.

Debate as to whether our patient has systemic Buerger's or Kohlmeier-Degos disease is largely of theoretical interest as both conditions are poorly understood; they may well be part of the same spectrum of vaso-occlusive diseases, or could be the endpoints of various vascular insults. Buerger's disease is diagnosed clinically, based on exclusion of other conditions. ${ }^{1}$ Kohlmeier-Degos disease is usually diagnosed based on typical porcelain-white atrophic papules ${ }^{5}$ which can also be seen in other conditions such as connective tissue diseases. ${ }^{10}$ The diagnosis of Kohlmeier-Degos disease is even more difficult in a person without skin lesions. Apart from smoking cessation for Buerger's disease, there are no known effective therapies. $^{11}$ It would be perilous to trial our patient on immunosuppressive therapy, given his recurrent Candida infections and a lack of evidence to support its use. Anticoagulation is contraindicated given his haemorrhage and it is reported as being ineffective in preventing thrombus formation in Buerger's disease. ${ }^{11}$

A small to medium vessel vasculitis such as polyarteritis nodosa is a differential diagnosis but on all three samples, there was only minimal vascular inflammation. In fact, inflammation was only seen in association with thrombosis and there was no fibrinoid necrosis. Moreover, his vasculitis screen was negative and the immunohistochemistry stains of the renal biopsy were also negative.
Another differential diagnosis for multi-system small to medium vessel thrombosis with absent or minimal vascular or perivascular inflammation is antiphospholipid syndrome. Our patient had negative antiphospholipid antibodies on multiple occasions, essentially ruling out this diagnosis. Previously our patient received warfarin therapy for 10 months after the initial diagnosis of renal failure but this did not prevent him from developing lower limb ischaemia, further detracting from the likelihood of an underlying coagulopathy.

Thrombotic microangiopathy due to thrombotic thrombocytopenic purpura (TTP) or atypical haemolytic uraemic syndrome (aHUS) was excluded clinically. He did not have hallmark features of microangiopathic haemolytic anaemia or thrombocytopenia and the episodic nature of his clinical course was not in keeping with TTP/aHUS. He also had normal coagulation profile, ruling out disseminated intravascular coagulation which could be triggered by systemic candidiasis.

Lastly, the uncertainty of the diagnosis makes the prospect of renal transplantation challenging. Little is known about the risk of a recurrent vaso-occlusive disease in the renal allograft. The low rate of renal involvement in these disorders suggests the risk of recurrence in the graft would be low; however, he would need to be clear of recurrent infections and have a prolonged period of disease quiescence prior to proceeding to transplantation. He has had no further clinically apparent thromboses for the last 6 months and continuing abstinence from tobacco smoking will be critical.

In summary, this report illustrates an extremely rare case of multisystem vaso-occlusive disorder. The diagnosis has been narrowed down to Buerger's disease or Kohlmeier-Degos 
disease. Arriving at a definitive diagnosis is not possible given the rarity of the presentation and the overlap of the two conditions. It will now be of interest to observe this patient's course with smoking cessation and future renal transplantation.

Conflicts of interest and sources of funding: The authors state that there are no conflicts of interest to disclose.

\author{
Shi Z. Choo ${ }^{1}$ \\ Ian Simpson ${ }^{2}$ \\ Moira J. Finlay ${ }^{3}$ \\ William R. Mulley ${ }^{1,4}$
}

${ }^{1}$ Department of Nephrology, and ${ }^{2}$ Department of Anatomical Pathology, Monash Health, Melbourne, ${ }^{3}$ Department of Anatomical Pathology, Royal Melbourne Hospital, Melbourne, and ${ }^{4}$ Centre for Inflammatory Diseases, Department of Medicine, Monash University, Clayton, Vic, Australia

Contact Dr Shi Zhou Choo.

E-mail: chooshizhou@hotmail.com

1. Shionoya S. Diagnostic criteria of Buerger's disease. Int J Cardiol 1998 66(Suppl 1): S243-5.

2. Tanaka K. Pathology and pathogenesis of Buerger's disease. Int $J$ Cardiol 1998; 66(Suppl 1): S237-42.

3. Fazeli B, Rezaee SA. A review on thromboangiitis obliterans pathophysiology: thrombosis and angiitis, which is to blame? Vascular 2011; 19: $141-53$.

4. Calguneri M, Ozturk MA, Ay H, et al. Buerger's disease with multisystem involvement. A case report and a review of the literature. Angiology 2004; 55: 325-8.

5. Theodoridis A, Makrantonaki E, Zouboulis CC. Malignant atrophic papulosis (Köhlmeier-Degos disease) - a review. Orphanet $J$ Rare Dis 2013; 8: 10

6. Scheinfeld N. Malignant atrophic papulosis. Clin Exp Dermatol 2007 32: $483-7$.

7. McFarland HR, Wood WG, Drowns BV, et al. Papulosis atrophicans maligna (Köhlmeier-Degos disease): a disseminated occlusive vasculopathy. Ann Neurol 1978; 3: 388-92.

8. Barcelli U, Crissman J, First MR, et al. Rapidly progressing arterioocclusive syndrome in a patient receiving long-term hemodialysis. Kohlmeier-Degos disease? Arch Intern Med 1981; 141: 1331-5.

9. Björck S, Johansson SL, Aurell M. Acute renal failure caused by a rapidly progressive arterio-occlusive syndrome-Kohlmeier-Degos disease? Scand J Urol Nephrol 1984; 18: 343-6.

10. Scheinfeld N. Degos' disease is probably a distinct entity: a review of clinical and laboratory evidence. J Am Acad Dermatol 2005; 52: 375-6.

11. Puéchal X, Fiessinger JN. Thromboangiitis obliterans or Buerger's disease: challenges for the rheumatologist. Rheumatology 2007; 46: 192-9.

DOI: http://dx.doi.org/10.1016/j.pathol.2017.06.008

\section{Atypical cutaneous melanocytic tumours arising in two patients with Li-Fraumeni syndrome}

Sir,

$\mathrm{Li}$-Fraumeni syndrome (LFS) is a rare cancer predisposition syndrome caused by a germline mutation of TP53 located on chromosome 17. This gene encodes a tumour suppressor protein involved in cell cycle regulation, apoptosis, DNA repair and cell senescence. This condition is well-known to be associated with soft tissue and bone sarcomas, brain tumours, adrenocortical carcinomas, leukaemias and breast cancers. These tumours can arise in early childhood and the risk subsists in adulthood. Few cutaneous melanomas have been described in this setting. We report two cases of atypical melanocytic cutaneous tumours arising in patients with LFS.

Case 1 was a 5-year-old girl who presented with a lumbar achromic nodule, measuring $7 \mathrm{~mm}$ in diameter, of recent rapid growth. She had a history of LFS discovered after the resection of an adrenocortical carcinoma at age 1 . She was recently diagnosed with an acute myeloid leukaemia. Histological examination showed an asymmetrical lesion with a lateral dermal nodular expansion (Fig. 1A). Otherwise, the lesion exhibited features of a conventional compound naevi with bland melanocytes. Melanocytes in the nodule displayed an epithelioid morphology with large oval nuclei and a wide amelanotic cytoplasm. Cytonuclear pleomorphism was present as well as mitotic activity reaching up to 4 mitoses $/ \mathrm{mm}^{2}$ in the hotspot area (Fig. 1B). No deep maturation or lymphocytic infiltrate were associated. Maximum tumour thickness reached $1.3 \mathrm{~mm}$. Immunohistochemistry showed strong p53 nuclear labelling of the epithelioid melanocytes restricted to the nodular area and, in contrast, weak focal expression in the lateral conventional component (Fig. 1C,D). The proliferative index was evaluated at $10 \%$ of nuclei with Ki-67/ MIB1 in the epithelioid nodular area and occasional cells in the compound naevus area. A BRAF V600E mutation was detected by VE1 antibody positivity throughout the lesion and later confirmed by DNA sequencing. Normal nuclear BAP1 labelling was found in all cells. Follow-up was uneventful regarding the skin lesion but the child died from the relapse of her leukaemia 18 months later.

Array comparative genomic hybridisation (CGH) performed on the nodule showed a near-haploid profile with monosomy of all chromosomes, excluding pairs 4, 7, 15, 20 and 21 (Fig. 1E). TP53 DNA sequencing found a p.R273H mutation (nucleotide substitution c. $818 \mathrm{G}>\mathrm{A}$ ) in exon 8 matching the known germline mutation.

Case 2 was a 27-year-old male patient with a history of LFS diagnosed after the occurrence of an adrenocortical carcinoma and a choroid plexus carcinoma during childhood and a neurinoma at age 14, who presented with an abdominal naevi. This heterogeneously pigmented, $7 \mathrm{~mm}$ lesion was removed and later sent to our institution for second opinion (Fig. 2A). Histological examination showed an asymmetric compound melanocytic proliferation. The junctional component displayed areas of large clustered nests of melanocytes in a hyperplasic and papillomatous epidermis alternating with areas containing only small nests or lentiginous melanocytes (Fig. 2B). In the upper dermis the melanocytes were arranged in scattered nests and isolated cells sometimes associated with a dense fibro-collagenic background. Melanocytes were of naevoid and epithelioid cytology with large cells, an often hyperchromatic or nucleolated nuclei with mild pleomorphism (Fig. 2C). No deep cytological or architectural maturation was seen. A few nests of hyperpigmented dermal melanocytes were present in the central portion of the lesion. Mitotic figures were scarce $\left(1 / \mathrm{mm}^{2}\right.$ at most). A mild perivascular lymphocytic infiltrate was intermingled with the lesion. Immunohistochemistry showed a strong p53 expression in both junctional and dermal melanocytes (Fig. 2D). Ki67/MIB1 stained less than 5\% of cells. BRAFVE1 stain was negative. 